J. Lake Sci.(湖泊科学), 2016, 28(4): 765-774

DOI 10. 18307/2016. 0409

(c) 2016 by Journal of Lake Sciences

\title{
鄱阳湖周溪湾沉积物中有机氯农药和多环芳烃的垂直分布特征
}

\author{
酣倩玉 ${ }^{1}$,赵中华 ${ }^{1 * *}$, 蒋 豫 $^{1,2}$, 张 路 $^{1}$ \\ (1: 中国科学院南京地理与湖泊研究所湖泊与环境国家重点实验室,南京 210008 ) \\ (2: 南京林业大学,南京 210037 )
}

\begin{abstract}
摘 要: 通过分析鄱阳湖周溪湾柱状沉积物中有机氯农药 (OCPs) 和多环芳烃 (PAHs) 的垂直分布特征, 初步探讨该区 OCPs 和 PAHs 的污染历史. 结果表明, 周溪湾柱状样中 OCPs 总含量范围在 $40.4 \sim 174.1 \mathrm{ng} / \mathrm{g}(\mathrm{dw})$ 之间, 六六六 $(\mathrm{HCHs})$ 是 其主要影响的化合物 $(16.5 \sim 153.6 \mathrm{ng} / \mathrm{g}(\mathrm{dw})$ ), 其次为氯丹类 (Chlordanes) 和滴滴涕类 (DDTs), 含量分别为 $3.4 \sim 44.0$ 和 $1.0 \sim 33.4 \mathrm{ng} / \mathrm{g}(\mathrm{dw})$. 垂直分布特征显示: 沉积相上 OCPs 的残留量比实际使用情况向后推迟 $10 \sim 20$ 年, 1950s $-1990 \mathrm{~s}$ OCPs 曾被大量使用, 到 2000 年左右在沉积物残留上达到顶峰, 随后残留量逐渐降低; 而近 20 年来, 该区已经鲜有新的 HCHs、Chlordanes 以及 DDTs 输人. PAHs 总含量范围为 $41.3 \sim 384 \mathrm{ng} / \mathrm{g}(\mathrm{dw})$, 芘和菲的含量最高, 分别为 $17.1 \sim 67.1$ 和 $2.68 \sim 139 \mathrm{ng} / \mathrm{g}(\mathrm{dw})$. 垂直分布特征显示, 1972 年以前, PAHs 总含量变幅不大, 然而近 10 年来 PAHs 的排放量急剧上升. 此外, 自 20 世纪末开始, 周溪湾区域 PAHs 的主要来源由以前的煤燃烧释放转化为交通污染排放, 并伴随有石油泄漏 情况.
\end{abstract}

关键词: 有机氯农药; 多环芳烃;沉积物; 垂直分布; 周溪湾; 鄱阳湖

\section{Vertical distribution characteristics of organochlorine pesticides and polycyclic aromatic hydrocarbons in a sedimentary core from Zhouxi Bay, Lake Poyang}

\author{
LI Qianyu ${ }^{1}$, ZHAO Zhonghua ${ }^{1 * *}$, JIANG $\mathrm{Yu}^{1,2} \&$ ZHANG Lu ${ }^{1}$ \\ (1: State Key Laboratory of Lake Science and Environment, Nanjing Institute of Geography and Limnology, Chinese Academy \\ of Sciences, Nanjing 210008, P.R. China) \\ (2: Nanjing Forestry University, Nanjing 210037, P.R.China)
}

\begin{abstract}
Zhouxi Bay from Lake Poyang is an important freshwater peals culture zone. A $72 \mathrm{~cm}$-deep sediment core was collected from the bay to examine the vertical distribution characteristics of organochlorine pesticides and polycyclic aromatic hydrocarbons. By measuring GC- $\mu$ ECD and HPLC-DAD/FLD, the pollution history of organochlorine pesticides and polycyclic aromatic hydrocarbons in this region was revealed. The total OCPs content ranged from 40.4 to $174.1 \mathrm{ng} / \mathrm{g}(\mathrm{dw})$. HCHs was the most abundant compound $(16.5-153.6 \mathrm{ng} / \mathrm{g}(\mathrm{dw}))$, followed by Chlordanes and DDTs, which were in the range of $3.4-44.0 \mathrm{ng} / \mathrm{g}(\mathrm{dw})$ and $1.0-33.4 \mathrm{ng} / \mathrm{g}(\mathrm{dw})$, respectively. The vertical distribution characteristics show that the residues of OCPs in sedimentary core were put off 10-20 years later than the usage of OCPs in environment. OCPs have been widely used since the mid-20th century, peaked in 2000, and then the residues of OCPs were gradually decreased. In the past 20 years, Limited amount of HCHs, Chlordanes and DDTs have entered in this area. The total PAHs content ranged from 41.3-384 ng/g( dw ). Among PAHs, the concentration of Pyr and Phe were 7.1-67.1 ng/g(dw) and 2.68-139 $\mathrm{ng} / \mathrm{g}(\mathrm{dw})$, respectively, much higher than other compounds. The vertical distribution characteristics show that the total PAHs content had a little variation before 1972, however, it has been sharply increased in last decades. Moreover, the sources of PAHs have changed from coal combustion to vehicle emission since the end of the 20th century, as well as oil spill.
\end{abstract}

Keywords: OCPs; PAHs; sediments; vertical distribution; Zhouxi Bay; Lake Poyang

* 国家重点基础研究发展计划 “973” 项目(2012CB417005)、科技部基础性工作专项(2012FY111800-03)、国家自然 科学基金青年基金项目 (41201535，41373017) 和江苏省自然科学基金项目 (BK2102503) 联合资助. 2015-02-01 收稿;2015 - 10 - 26 收修改稿. 畉倩玉 (1990 ) ,女, 硕士研究生;E-mail : ddandpp0857@ 163.com.

** 通信作者; E-mail: zhzhao@ niglas.ac.cn. 
有机氯农药 (Organochlorine Pesticides, OCPs) 是一种人工合成的有机物, 其理化性质稳定, 难以降解, 使 得这类物质在 1982 年被全面禁止使用后仍在土壤、沉积物和农作物中尚有大量残留 ${ }^{[1-3]}$. 多环芳烃 (Polycyclic Aromatic Hydrocarbons,PAHs) 主要来源于人类活动而引起的不完全燃烧, 如炼焦、煤炭及石油燃 烧、垃圾焚烧和交通排放等 ${ }^{[4-7]}$. 沉积物是湖泊生态系统的重要组成部分, 由于 OCPs 和 PAHs 具有亲脂疏水 的特性, 使得它们进入湖泊后易吸附存留于沉积物有机质中. 因此, 研究湖泊沉积物中 OCPs 和 PAHs 的残 留污染状况具有特别重要的意义.

鄱阳湖是我国第一大淡水湖泊,其流域包括赣江、抗河、信江、饶河、修水 5 大支流流域, 占江西全流域 面积的 $96.8 \%$, 几乎汇集江西省内的各种水污染物 ${ }^{[8]}$. 然而以往对鄱阳湖流域水体的水质监测数据仅局限 于常规的水化学指标, 如总氮、总磷、化学需氧量等; 有关 OCPs 和 PAHs 的污染状况, 自 1990s 以来虽曾作过 一些调查和研究, 但主要集中于鄱阳湖中心湖区 ${ }^{[9]}$ 和南边洲滩地区 ${ }^{[10-11]}$, 周溪湾地区尚未见详细报道. 周溪 湾位于都昌县的东南部, 鄱阳湖东北岸, 是典型的边缘深水区域, 丰水期水位上涨与鄱阳湖大水面相连, 汇 成一体; 枯水期水位下降, 形成断流成为封闭式水体, 相对闭塞. 此外, 周溪地区也是我国重要的淡水珍珠养 殖基地 ${ }^{[12]}, 2007$ 年 6 月 1 日 《扬州日报》曾报导过该区域因养殖珍珠而滥施化肥和农药,污水排出未经处理 则直接人湖, 导致水质变差. 报导后虽有专家做过关于周溪湾富营养化情况的调查, 但有机污染方面却并未 有所研究. 因此, 本文选定鄱阳湖周溪湾为研究区, 采集 1 根湖泊沉积物剖面, 对其中的 OCPs 和 PAHs 含量 进行分析测定, 初步探讨这两类有机污染物在周溪湾柱状沉积物中的污染状况和垂向的年度分布特征, 为 进一步研究鄱阳湖的生态环境安全提供科学依据.

\section{1 材料与方法}

\section{1 样品采集}

2011 年 11 月选定鄱阳湖周溪湾区域为采样点 $\left(29^{\circ} 09^{\prime} \mathrm{N}, 116^{\circ} 22^{\prime} \mathrm{E}\right)$, 枯水期湖泊水面下降, 在接近水面 的河床或裸露的三角洲水陆相连的区域, 利用有机玻璃管插人箱式重力采样器采集 1 根总长为 $72 \mathrm{~cm}$ 的柱 状沉积物样品. 随后将采集到的样品用分样器按每 $2 \mathrm{~cm}$ 厚度分样, 并装人自封袋中冷冻保存送回实验室进 行预处理和分析,共分得 36 个样品, 编号自上而下依次为 ZX1 ZX36.

\section{2 材料与试剂}

20 种有机氯农药混合标样品 $\alpha$-六六六 $(\alpha-\mathrm{HCH}) 、 \beta$-六六六 $(\beta-\mathrm{HCH}) 、 \gamma$-六六六 $(\gamma-\mathrm{HCH})$ 、 $\delta$-六六六 $(\delta$ $\mathrm{HCH}$ )、反式-氯丹 $(\alpha$-Chlordane) 、顺式-氯丹 $(\gamma$-Chlordane)、艾氏剂 (Aldrin)、狄氏剂 (Dieldrin)、硫丹 I ( Endosulfan I)、硫丹 II (Endosulfan II)、硫丹硫酸盐(Endosulfan sulfate)、异狄氏剂( Endrin)、异狄氏剂醛( Endrin aldehyde)、异狄氏剂酮( Endrin ketone)、七氯( Heptachlor)、七氯环氧化物 (Heptachlor epoxide)、甲氧滴滴涕 (Methoxychlor)、p, $\mathrm{p}^{\prime}$-DDT、p, $\mathrm{p}^{\prime}$-DDE、p, $\mathrm{p}^{\prime}$-DDD, 以及 16 种美国 EPA 优先控制 PAHs 的混合标样荎 $(\mathrm{NaP})$ 、

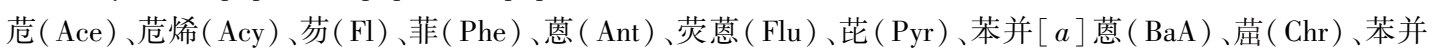
$[b]$ 苂葱 $(\mathrm{BbF})$ 、苯并 $[k]$ 苂蒽 $(\mathrm{BkF})$ 、苯并 $[a]$ 芘 $(\mathrm{BaP})$ 、二苯并 $[a, h]$ 萝 $(\mathrm{DahA}) 、$ 、苯并 $[g, h, i]$ 萠 $(\mathrm{BghiP}) 、$ 狮并 $[1,2,3-c, d]$ 萠 (IP), 均购自于美国 Supelco 公司. 其他主要试剂正己烷、二氯甲烷、甲醇、乙腈等为色谱 纯级别. 层析硅胶则经甲醇和二氯甲烷超声清洗 $20 \mathrm{~min}$ 后 $180^{\circ} \mathrm{C}$ 活化 $12 \mathrm{~h}$; 中性氧化铝同样经超声后置于 $250^{\circ} \mathrm{C}$ 马弗炉中活化 $12 \mathrm{~h}$ 待用. 无水硫酸钠和石英砂为分析纯, 马弗炉 $450^{\circ} \mathrm{C}$ 焙烧 $6 \mathrm{~h}$ 后于干燥器内保存.

\section{3 样品处理}

将采集到的底泥样品冷冻干燥后, 分别研磨过 100 目篮. 称取 $5 \mathrm{~g}$ 左右的样品粉末, 加人 $8 \mathrm{~g}$ 左右的石英 砂和 $3 \mathrm{~g}$ 铜粉, 充分摇匀后装人加速溶剂萃取仪 (Accelerated solvent extraction system, ASE, Dionex 100) 的萃 取池中进行高温高压萃取. 萃取所用溶剂为色谱纯二氯甲烷, 加热温度为 $100^{\circ} \mathrm{C}$, 压力 $1500 \mathrm{psi}$, 每个样品静 态提取 2 次, 每次 $10 \mathrm{~min}$. 将萃取完的溶液转移至蒸发瓶内, 通过旋转蒸发仪旋转蒸发至近干, 加人 $5 \mathrm{ml}$ 正 己烷为置换溶剂, 继续浓缩至 $1 \mathrm{ml}$ 左右转移至 $5 \mathrm{ml}$ 玻璃刻度量管中. 再将浓缩液通过硅胶: 氧化铝体积分 数比为 $2: 1$ 的玻璃层析柱, 用 $15 \mathrm{ml}$ 正己烷和 $70 \mathrm{ml}$ 正己烷:二氯甲烷体积分数比为 $7: 3$ 的混合溶液依次淋洗 有机组分, 收集正己烷: 二氯甲烷组分淋洗液并再次旋蒸浓缩, 正己烷溶剂置换至 $5 \mathrm{ml}$, 最后氮吹仪柔和氮 吹定容到 $0.5 \mathrm{ml}$ 转移至 $2 \mathrm{ml}$ 气相小瓶内, $-20^{\circ} \mathrm{C}$ 保存待仪器分析. 


\section{4 样品分析}

OCPs 检测采用 Agilent 7890A 气相色谱仪, 配以 ${ }^{63} \mathrm{Ni}$ 电子捕获检测器 (GC- $\mu$ ECD ). HP-5MS 毛细管色谱 柱 $(30 \mathrm{~m} \times 0.25 \mathrm{~mm} \times 0.25 \mu \mathrm{m}, \mathrm{J} \& \mathrm{~W})$, 载气为高纯氦气, 柱流量为 $1.5 \mathrm{ml} / \mathrm{min}$, 进样量为 $1.0 \mu \mathrm{l}$, 非分流进样, 尾吹高纯氮气, $60 \mathrm{ml} / \mathrm{min}$. 柱升温程序为: 初温为 $60^{\circ} \mathrm{C}$, 以 $10^{\circ} \mathrm{C} / \mathrm{min}$ 升至 $170^{\circ} \mathrm{C}$, 保持 $2 \mathrm{~min}$; 以 $5^{\circ} \mathrm{C} / \mathrm{min}$ 升至 $280^{\circ} \mathrm{C}$, 保持 $3 \mathrm{~min}$; 最后以 $15^{\circ} \mathrm{C} / \mathrm{min}$ 升至 $300^{\circ} \mathrm{C}$. 汽化室和检测器的温度分别为 $250^{\circ} \mathrm{C}$ 和 $320^{\circ} \mathrm{C}$. $\mathrm{PAHs}$ 检测 采用 Agilent 1200 高效液相色谱仪 (HPLC) 完成, 以二极管阵列紫外检测器 (DAD) 串联荧光检测器 (FLD) 进 行 PAHs 的定性和定量分析, 自动进样器. DAD 检测器波长设定为 $254 \mathrm{~nm}$, FLD 检测器激发 $\left(E_{\mathrm{x}}\right)$ 和发射 $\left(E_{\mathrm{m}}\right)$ 波长条件根据时间而改变: 初始 $E_{\mathrm{x}} / E_{\mathrm{m}}: 260 \mathrm{~nm} / 280 \mathrm{~nm} ; 2 \mathrm{~min}$ 后 $E_{\mathrm{x}} / E_{\mathrm{m}}: 280 \mathrm{~nm} / 330 \mathrm{~nm} ; 9.2 \mathrm{~min}$ 后 $E_{\mathrm{x}} / E_{\mathrm{m}}: 280 \mathrm{~nm} / 380 \mathrm{~nm} ; 14 \min$ 后 $E_{\mathrm{x}} / E_{\mathrm{m}}: 260 \mathrm{~nm} / 450 \mathrm{~nm} ; 16.5 \min$ 后 $E_{\mathrm{x}} / E_{\mathrm{m}}: 260 \mathrm{~nm} / 380 \mathrm{~nm} ; 28 \min$ 后 $E_{\mathrm{x}} / E_{\mathrm{m}}: 290 \mathrm{~nm} / 410 \mathrm{~nm} ; 35.5 \mathrm{~min}$ 后 $E_{\mathrm{x}} / E_{\mathrm{m}}: 290 \mathrm{~nm} / 500 \mathrm{~nm}$. 色谱柱为 PAHs Waters C18 $(4.6 \times 250 \mathrm{~mm}$, 粒 径: $5 \mu \mathrm{m}$, Waters, Japan), 流动相为乙腈水溶液, 进样量为 $20 \mu \mathrm{l}$, 柱温为 $25^{\circ} \mathrm{C}$, 梯度洗脱, 洗脱条件: $0 \sim 15$ $\min , 60 \%$ 乙腈+40\%纯水; $15 \sim 30 \mathrm{~min}, 60 \% \sim 100 \%$ 乙腈; $30 \sim 40 \mathrm{~min}, 100 \%$ 乙腈; $40 \sim 40.1 \mathrm{~min}, 100 \% \sim 60 \%$ 乙 腈; 停止时间为 $48 \mathrm{~min}$.

\section{5 质量控制和回收率}

依据标准物质的出峰顺序和保留时间对 OCPs 和 PAHs 定性, 采用 6 点校正曲线峰面积外标法定量, 其 中 OCPs 的定量同时采用内标法进行 (内标化合物为 2,4,5,6-四氯间二甲苯和十氯联苯). 为了保证实验分 析数据的准确性和可靠性,每次样品分析的过程中均同步设置方法空白实验和基质加标实验判定整个实验 操作过程中是否有基质的干扰. 方法回收率实验选用经马弗炉焙烧处理过的石英砂代替环境样品, 加人定 量的 PAHs 或 OCPs 标准化合物, 按照上述同样的预处理方法进行样品处理, 仪器分析测定每种化合物的方 法回收率. 方法检测限以基质样品中能够产生 3 倍信噪比 $(\mathrm{S} / \mathrm{N})$ 的样品量确定. 实验表明, HPLC-DAD/FLD 测定 16 种优控 PAHs 的方法回收率为 76\% 108\%, 方法检测限为 $0.09 \sim 3.52 \mathrm{ng} / \mathrm{g}$; GC- $\mu$ ECD 测定 OCPs 的 方法回收率为 $63 \% \sim 115 \%$, 方法检测限为 $0.01 \sim 1.55 \mathrm{ng} / \mathrm{g}$, 满足美国环境保护总局 (USEPA) 环境样品质量 分析要求.

\section{6 数据分析}

所有样品中 OCPs 和 PAHs 的浓度均以干重法计算 $(\mathrm{ng} / \mathrm{g}(\mathrm{dw}))$, 低于检测限和未检出的化合物浓度, 不 予计算. 数据分析借助 SPSS 20.0 for windows 以及 Origin 8.5 软件进行. PAHs 的来源解析利用 SPSS 的主成 分分析软件包和多元线性回归软件包. 进行主成分分析时, 其检验方式 Kaiser-Meyer-Olkin (KMO) 的值如大 于 $0.5, P$ 值小于 0.05 , 则代表适合做主成分分析, 即选取特征值大于 1 的因子解析. 回归分析借助 Origin 8.5 软件进行线性拟合, 其余 OCPs 和 PAHs 的比值以及相关成图皆是借用 Origin 8.5 软件完成.

\section{2 结果与讨论}

\section{1 柱状沉积物样 OCPs 的垂直分布特征}

2.1.1 OCPs 含量与垂直分布 本次样品中, 20 种 OCPs 化合物均有检出, 测得的 OCPs 总含量介于 40.4 $174.1 \mathrm{ng} / \mathrm{g}(\mathrm{dw})$ 之间, 平均值为 $104.1 \mathrm{ng} / \mathrm{g}(\mathrm{dw})$, 最高含量出现在 ZX6 (10 12 cm) 样品中, 最低含量在 ZX7 $(12 \sim 14 \mathrm{~cm})$ 样品. 除去 Heptachlor、 $\alpha$-Chlordane、Endosulfan I、Endosulfan II 和 p, p'-DDD, 其余化合物在所有 样品中的检出率均为 $100 \%$ (表 1). HCHs (包含 $\alpha, \beta, \gamma, \delta$ - HCH) 的检出含量介于 $16.5 \sim 153.6 \mathrm{ng} / \mathrm{g}(\mathrm{dw}$ ) 之间 (平均为 $57.3 \mathrm{ng} / \mathrm{g}(\mathrm{dw})$ ), 其次为 Chlordanes (包含 Heptachlor, Heptachlor epoxide, $\alpha$-Chlordane 以及 $\gamma$ Chlordane) 和 DDTs (包含 $\mathrm{p}, \mathrm{p}^{\prime}$-DDD, p, $\mathrm{p}^{\prime}$-DDE 以及 $\mathrm{p}, \mathrm{p}^{\prime}$-DDT), 含量范围分别在 3.4 44.0 $\mathrm{ng} / \mathrm{g}(\mathrm{dw}$ ) 之间 (平均为 $16.4 \mathrm{ng} / \mathrm{g}(\mathrm{dw}$ ) ) 和 $1.0 \sim 33.4 \mathrm{ng} / \mathrm{g}(\mathrm{dw}$ ) 之间 (平均为 $9.1 \mathrm{ng} / \mathrm{g}(\mathrm{dw}$ ) ). HCHs 的 4 种异构体中 $\beta-\mathrm{HCH}$ 的检出含量最高 $(4.3 \sim 73.4 \mathrm{ng} / \mathrm{g}(\mathrm{dw})$, 平均为 $33.9 \mathrm{ng} / \mathrm{g}(\mathrm{dw}))$, 其次为 $\delta-\mathrm{HCH}(0.07 \sim 145.8 \mathrm{ng} / \mathrm{g}(\mathrm{dw})$, 平均 为 $13.6 \mathrm{ng} / \mathrm{g}(\mathrm{dw}))$; DDTs 的主要检出物是 $\mathrm{p}, \mathrm{p}^{\prime}-\mathrm{DDE}(1.0 \sim 13.4 \mathrm{ng} / \mathrm{g}(\mathrm{dw})$, 平均为 $5.9 \mathrm{ng} / \mathrm{g}(\mathrm{dw}))$ 和 $\mathrm{p}, \mathrm{p}^{\prime}-$ DDD ( ND 19.3 ng/g $(\mathrm{dw})$, 平均 $3.2 \mathrm{ng} / \mathrm{g}(\mathrm{dw}))$. 周溪湾表层沉积物样品 (ZX1-2) 的 OCPs、HCHs 和 Chlordanes 的含量与全国以及鄱阳湖流域其他地区相比总体偏高, 但 DDTs 的含量却相对较低(表 2). 
表 1 周溪湾柱状沉积物中 OCP 化合物检测结果

Tab.1 Detection profiles of individual OCP compounds in sedimentary Zhouxi Bay core

\begin{tabular}{lcccc}
\hline OCP 化合物 & $\begin{array}{c}\text { 最小值/ } \\
(\mathrm{ng} / \mathrm{g}(\mathrm{dw}))\end{array}$ & $\begin{array}{c}\text { 最大值/ } \\
(\mathrm{ng} / \mathrm{g}(\mathrm{dw}))\end{array}$ & $\begin{array}{c}\text { 平均值士标准差/ } \\
(\mathrm{ng} / \mathrm{g}(\mathrm{dw}))\end{array}$ & $\begin{array}{c}\text { 检出率/ } \\
\%\end{array}$ \\
\hline$\alpha$-HCH & 0.8 & 18.0 & $57.3 \pm 4.3$ & 100.0 \\
$\beta$-HCH & 4.3 & 73.4 & $33.9 \pm 20.7$ & 100.0 \\
$\gamma$-HCH & 0.4 & 4.5 & $2.5 \pm 1.2$ & 100.0 \\
$\delta$-HCH & 0.07 & 145.8 & $13.6 \pm 29.8$ & 100.0 \\
Heptachlor & $\mathrm{nd}$ & 23.4 & $3.9 \pm 4.5$ & 75.0 \\
Aldrin & 0.5 & 0.8 & $0.6 \pm 0.1$ & 100.0 \\
Heptachlor epoxide & 0.7 & 20.1 & $7.5 \pm 3.2$ & 100.0 \\
$\gamma$-Chlordane & 1.5 & 21.9 & $3.2 \pm 3.3$ & 100.0 \\
Endosulfan I & $\mathrm{nd}$ & 2.4 & $1.1 \pm 0.9$ & 63.9 \\
$\alpha$-Chlordane & $\mathrm{nd}$ & 3.2 & $1.8 \pm 0.7$ & 97.2 \\
Endrin & 0.01 & 0.3 & $0.1 \pm 0.1$ & 100.0 \\
Dieldrin & 0.01 & 0.3 & $0.1 \pm 0.1$ & 100.0 \\
$\mathrm{p}, \mathrm{p}^{\prime}$-DDE & 0.1 & 13.4 & $5.9 \pm 3.3$ & 100.0 \\
Endosulfan II & $\mathrm{nd}$ & 17.6 & $5.4 \pm 4.8$ & 97.2 \\
p,p'-DDD & $\mathrm{nd}$ & 20.0 & $8.0 \pm 6.2$ & 61.1 \\
Endrin aldehyde & 0.2 & 3.3 & $1.4 \pm 1.0$ & 100.0 \\
Endosulfan sulfate & 0.2 & 10.9 & $6.3 \pm 3.5$ & 100.0 \\
$\mathrm{p}, \mathrm{p}^{\prime}$-DDT & 0.0004 & 0.2 & $0.05 \pm 0.1$ & 100.0 \\
Endrin ketone & 0.3 & 9.8 & $4.1 \pm 2.8$ & 100.0 \\
Methoxychlor & 0.2 & 4.5 & $2.1 \pm 1.2$ & 100.0 \\
\hline
\end{tabular}

nd 表示未检测出.
将此次周溪湾柱状样品按每 $5 \mathrm{~cm}$ 划分进行 ${ }^{210} \mathrm{~Pb}$ 测年, 得出分层样品的大 致沉积年代. 根据沉积年代, 该区沉积物 中 OCPs 含量的垂直分布情况表明, 1950 年以前, 沉积物中 OCPs 含量总体波动较 大, 且含量偏高 $(121.7 \pm 25.6 \mathrm{ng} / \mathrm{g}(\mathrm{dw})$ (图 1). 我国从 1940s 开始, 才渐渐生产 并使用 OCPs, 但在 1940s - 1950s 之前沉 积物中就已检测出高含量的 OCPs, 这可 能是由于全球长距离的迁移所带来的. 据悉欧洲等发达国家在 1940s 之前就已 大规模使用 OCPs, 而其中的 Aldrins 类农 药并未在中国生产和使用过, 却在早期 的沉积物样品中检测出 Aldrins 含量, 说 明此时的沉积物污染主要是由于 OCPs 的 持久和挥发特性通过大气和水循环长距 离的输人到鄱阳湖乃至周溪湾地区 ${ }^{[11]}$. 1950-1990 年之间, OCPs 含量总体波动 不大, 且相对偏低 $(98.2 \pm 18.5 \mathrm{ng} / \mathrm{g}(\mathrm{dw})$. $1940 \mathrm{~s}-1950 \mathrm{~s}$ 中国开始生产 OCPs, 到 $1960 \mathrm{~s}-1980 \mathrm{~s}$ 是中国大量使用和生产 OCPs 的黄金 20 年, 如 HCH、DDT、毒杀 芬、六氯苯、氯丹和七氯. 据统计, 20 世纪 中末期中国共生产 $490 \times 10^{4} \mathrm{t} \mathrm{HCH}$ 和 $40 \times 10^{4} \mathrm{t}$ DDT, 分别占国际用量的 $33 \%$ 和

$20 \%{ }^{[16]}$, 在 1980s 达到顶峰. 随后, 在 1982 年开始实施农药登记和记录, 并逐渐先后禁止了毒杀芬、氯丹和 七氯的使用, 只保留了少量的 DDT 和六氯苯的登记和生产, OCPs 使用量迅速减少 ${ }^{[18]}$. 然而这种使用情况的 变化反映在沉积物中 OCPs 的残留量的变化, 亦对应了一个明显的异常增高再迅速降低的过程, 只是在沉积 相上显示比使用时期向后推迟约 10 20 年, 1990-2000 年左右表现出 OCPs 残留量的高峰 $(93.5 \pm$ $54.2 \mathrm{ng} / \mathrm{g}(\mathrm{dw}))$, 存在一定的延迟沉降性传输延迟. 2000 年以后, OCPs 残留量恢复到以往水平, 但近十几年 又有逐渐增加的趋势.

表 2 不同地区表层沉积物中 OCPs 含量 $(n g / g(d w))$

Tab.2 OCPs concentrations in surface sediments from different regions

\begin{tabular}{|c|c|c|c|c|c|c|c|c|}
\hline \multirow{2}{*}{ 来源水体 } & \multicolumn{2}{|c|}{ OCPs } & \multicolumn{2}{|c|}{$\mathrm{HCHs}$} & \multicolumn{2}{|c|}{ DDTs } & \multicolumn{2}{|c|}{ Chlordanes } \\
\hline & 范围 & 平均值 & 范围 & 平均值 & 范围 & 平均值 & 范围 & 平均值 \\
\hline 辽河 [13] & $0.2 \sim 7.5$ & 1.2 & $0.2 \sim 1.1$ & 0.4 & $0.01 \sim 7.0$ & 1.0 & $-1)$ & - \\
\hline 汾河 [14] & $2.2 \sim 25.2$ & 6.3 & $0.5 \sim 7.3$ & 1.7 & nd $~ 19.3$ & 1.8 & nd $\sim 2.7$ & 1.0 \\
\hline 巢湖 [15] & $0.9 \sim 36.9$ & 13.7 & $0.2 \sim 1.8$ & 0.8 & $0.3 \sim 31.0$ & 4.4 & - & - \\
\hline 太湖 [16] & $4.2 \sim 461$ & 73.0 & $0.07 \sim 5.8$ & 1.7 & $0.3 \sim 375$ & 53.9 & - & - \\
\hline 鄱阳湖湖心区 ${ }^{[9]}$ & $12.9 \sim 55.7$ & 35.8 & $0.5 \sim 6.9$ & 3.0 & $0.8 \sim 48.7$ & 25.0 & - & - \\
\hline 鄱阳湖龙口地区 ${ }^{[17]}$ & $7.8 \sim 93.7$ & 37.4 & $0.7 \sim 39.6$ & 9.0 & $2.4 \sim 49.4$ & 22.9 & nd $\sim 4.4$ & 1.4 \\
\hline 鄱阳湖周溪湾(本研究) & $210.4 \sim 433.5$ & 322.0 & $28.4 \sim 58.1$ & 43.2 & $1.0 \sim 1.6$ & 1.3 & $8.2 \sim 9.0$ & 8.6 \\
\hline
\end{tabular}

1 ) 文献中未给出. 


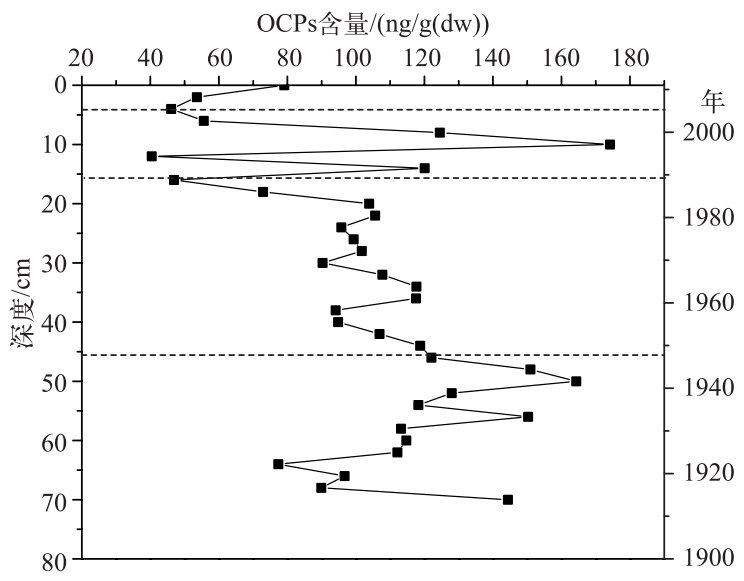

图 1 周溪湾柱状沉积物 OCPs 含量垂直分布特征(虚线代表时间段的分界线)

Fig.1 Vertical distribution characteristics of OCPs contents in sedimentary Zhouxi Bay core

2.1.2 OCPs 组分及来源特征工业纯 $\mathrm{HCH}$ 和林丹的使用是环境中 $\mathrm{HCHs}$ 的直接来源. $\mathrm{HCHs}$ 四种异构体 $(\alpha$, $\beta, \gamma, \delta-\mathrm{HCH})$ 的比值可以用来反映 HCH 杀虫剂的使用情况. 一般而言, 当 $\alpha-/ \gamma-\mathrm{HCH}$ 比值介于 $3 \sim 7$ 时, 表示来 源于工业纯 $\mathrm{HCH}$, 并可能经过大气长距离运输; 而当 $\alpha-/ \gamma-\mathrm{HCH}$ 的比值小于 3 时, 表示周围环境中林丹代替了 工业纯 $\mathrm{HCH}$ 在使用; 若 $\alpha-/ \gamma$-HCH 比值接近于 1 , 则表示该区又有新的 $\gamma$-HCH 的输人. $\beta$ - $\mathrm{HCH}$ 在 HCHs 中结构 最为稳定, 所以高比例的 $\beta$ - $\mathrm{HCH}$ 往往意味着 $\mathrm{HCHs}$ 在环境中存在的越久 ${ }^{[19]}$. 由于 $\alpha-\mathrm{HCH}$ 比 $\gamma$ - $\mathrm{HCH}$ 更难降解, 导致其在沉积物中埋藏年代越久, $\alpha-/ \gamma-\mathrm{HCH}$ 值则越大, 但这并不意味环境中 $\mathrm{HCHs}$ 的来源就属工业纯 $\mathrm{HCH}$, 因此越接近表层的沉积样品其 $\alpha-/ \gamma-\mathrm{HCH}$ 的值越能反映 HCHs 的实际使用情况 (图 2a). 1960-1995 年 $\alpha-/ \gamma$ $\mathrm{HCH}$ 的值基本介于 $3 \sim 7$ 之间, 说明这段时期内工业纯 $\mathrm{HCH}$ 在周溪湾地区作为主要杀虫剂而广泛使用. 自 1990 年以后, $\alpha-/ \gamma$-HCH 的值开始小于 3 , 个别时间段 (1995-2000 年) 比值接近于 1 (图 2a), 说明禁止使用工 业纯 $\mathrm{HCH}$ 后, 林丹开始作为替代品在该区使用, 同时不完全排除在特定时段内有新的 $\gamma-\mathrm{HCH}$ 输人. $\beta-\mathrm{HCH}$ 在 所有样品以及表层样品中所占 $\mathrm{HCHs}$ 比例最大,分别为 $59.1 \%$ 和 $82.0 \%$, 这意味着周溪湾地区的 HCHs 基本都 已经转变为最为稳定和难以降解的 $\beta-\mathrm{HCH}$,且最近几年该区亦没有或少有新的 $\mathrm{HCHs}$ 的输人.
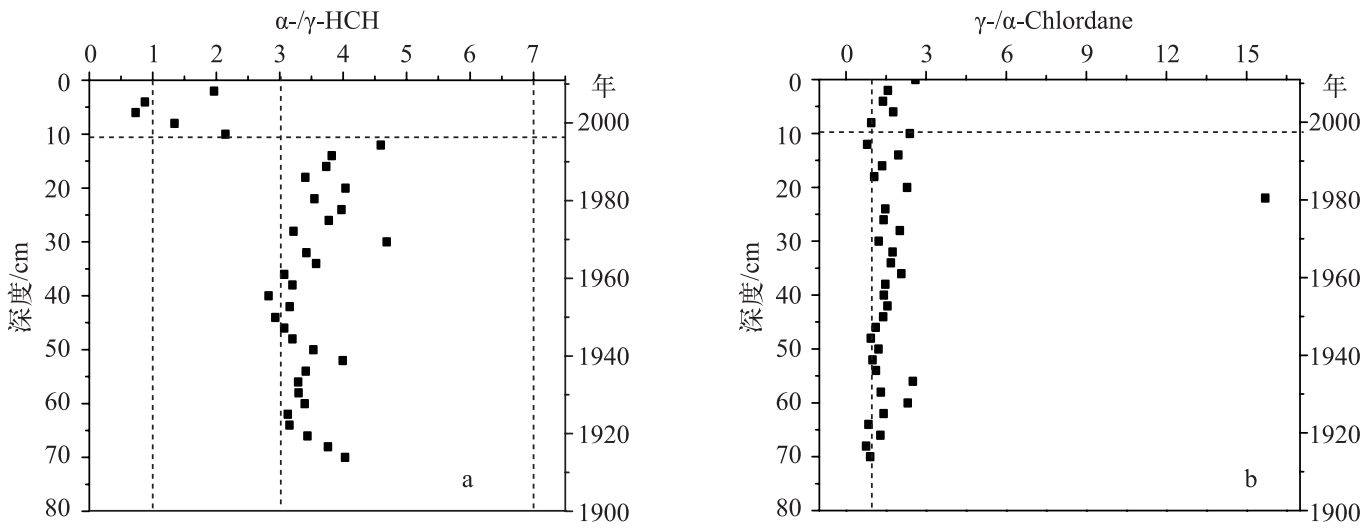

图 2 周溪湾柱状沉积物 $\alpha-/ \gamma-\mathrm{HCH}$ (a) 及 $\gamma-/ \alpha$-Chlordane (b) 的比值(虚线代表比值分界线)

Fig. 2 Ratio of $\alpha-\mathrm{HCH} / \gamma-\mathrm{HCH}$ (a) and $\gamma-/ \alpha$-Chlordane(b) in sedimentary Zhouxi Bay core

工业 Chlordane 是一种混合成分超过 140 多种化合物的有机氯农药, 被广泛用于杀虫剂和除草剂当 中 ${ }^{[20]}$. 其中工业 Chlordane 最主要的成分包含 $13 \%$ 的 $\alpha$-Chlordane、 $11 \%$ 的 $\gamma$-Chlordane 以及 5\% 的 Heptachlor. 由于 $\alpha$-Chlordane 比 $\gamma$-Chlordane 在环境中更易降解, 因此 $\gamma$-/ $\alpha$-Chlordane $>1.0$ 则意味着工业 Chlordane 在环 
境中存在久远 ${ }^{[21-22]}$. 接近表层的样品中 $\gamma-/ \alpha$-Chlordane 的值绝大部分大于 1.0 , 说明周溪湾地区目前已没有 或少有新的工业 Chlordane 的输人 (图 2b). 此外, Heptachlor 的降解物 Heptachlor epoxide 的检测含量是其母 体化合物的 2 倍,亦表示了该区的工业 Chlordane 在环境中存在时间久远.

$p, p^{\prime}$-DDT 是工业纯 DDT 的主要成分, 并且 $p, p^{\prime}$-DDT 在戻氧条件下会代谢为 $p, p^{\prime}$-DDD, 而在好氧条件 下则会代谢为 $\mathrm{p}, \mathrm{p}^{\prime}-\mathrm{DDE}$. 因此, 研究常用 $\left(\mathrm{p}, \mathrm{p}^{\prime}-\mathrm{DDE}+\mathrm{p}, \mathrm{p}^{\prime}-\mathrm{DDD}\right) / \sum \operatorname{DDT} \mathrm{s}\left(\mathrm{p}, \mathrm{p}^{\prime}-\mathrm{DDT}\right.$ 与降解产物之和) 和 $\mathrm{p}, \mathrm{p}^{\prime}-\mathrm{DDD} / \mathrm{p}, \mathrm{p}^{\prime}-\mathrm{DDE}$ 的比值来判定某一地区是否有新的 DDT 输人以及 DDT 的降解环境. 本次 36 个柱状沉 积物样品中, $\left(\mathrm{p}, \mathrm{p}^{\prime}-\mathrm{DDE}+\mathrm{p}, \mathrm{p}^{\prime}-\mathrm{DDD}\right) / \sum \mathrm{DDT}$ 值均超过 0.99 , 说明周溪湾地区已没有新的 DDT 的输人, 并且 DDT 系列农药大部分已经转换为稳定的降解物质. $\mathrm{p}, \mathrm{p}^{\prime}-\mathrm{DDD} / \mathrm{p}, \mathrm{p}^{\prime}-\mathrm{DDE}$ 的比值有一个明显的转换: 接近底 部的沉积物样品即 ZX28 ZX36 (54 72 cm) p, $\mathrm{p}^{\prime}-\mathrm{DDD} / \mathrm{p}, \mathrm{p}^{\prime}-\mathrm{DDE}>1$, 说明这部分 DDT 的降解环境为厌氧,

表 3 周溪湾柱状沉积物中 PAH 化合物检测结果

Tab.3 Detection profiles of individual PAH compounds in sedimentary Zhouxi Bay core

\begin{tabular}{cccc}
\hline $\begin{array}{c}\text { PAH } \\
\text { 化合物 }\end{array}$ & $\begin{array}{c}\text { 最小值/ } \\
(\mathrm{ng} / \mathrm{g}(\mathrm{dw}))\end{array}$ & $\begin{array}{c}\text { 最大值/ } \\
(\mathrm{ng} / \mathrm{g}(\mathrm{dw}))\end{array}$ & $\begin{array}{c}\text { 平均值士标准差/ } \\
(\mathrm{ng} / \mathrm{g}(\mathrm{dw}))\end{array}$ \\
\hline Nap & 0.6 & 31.9 & $6.2 \pm 6.6$ \\
Acy & 1.6 & 97.3 & $5.3 \pm 16.1$ \\
$\mathrm{Fl}$ & 2.7 & 31.2 & $8.0 \pm 5.2$ \\
Phe & 2.7 & 139.2 & $15.0 \pm 24.2$ \\
Ant & 3.6 & 34.4 & $11.7 \pm 6.6$ \\
Pyr & 17.1 & 67.1 & $32.5 \pm 13.0$ \\
$\mathrm{Chr}$ & 0.06 & 1.4 & $0.4 \pm 0.4$ \\
$\mathrm{BbF}$ & 1.5 & 23.6 & $6.4 \pm 5.1$ \\
$\mathrm{BkF}$ & 0.7 & 7.6 & $1.6 \pm 1.4$ \\
$\mathrm{BaP}$ & 0.03 & 2.3 & $0.3 \pm 0.5$ \\
DahA & 0.2 & 6.4 & $1.3 \pm 1.2$ \\
BghiP & 0.2 & 3.9 & $0.7 \pm 0.8$ \\
\hline
\end{tabular}

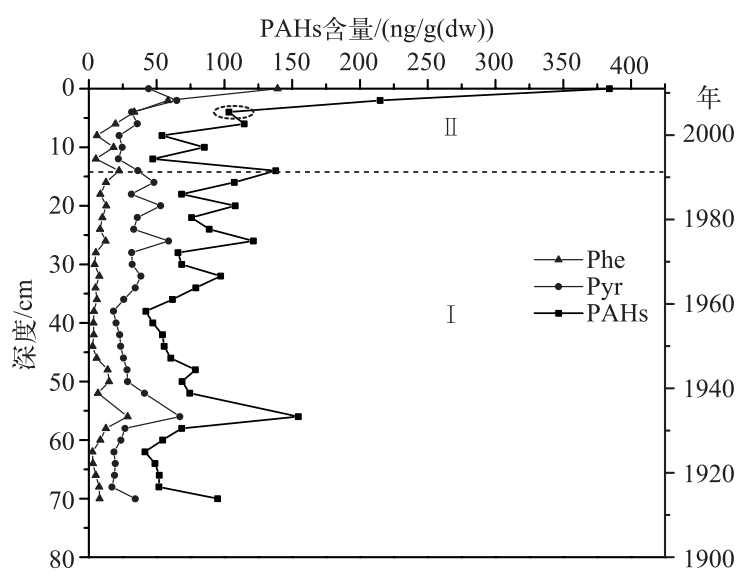

图 3 周溪湾柱状沉积物 PAHs 含量垂直分布 (虚线代表时间分界线)

Fig.3 Vertical distribution of PAHs contents in sedimentary of Zhouxi Bay core
而上层沉积物的 $\mathrm{p}, \mathrm{p}^{\prime}-\mathrm{DDD} / \mathrm{p}, \mathrm{p}^{\prime}-\mathrm{DDE}<1$, 并且在部 分样品中 $\mathrm{p}, \mathrm{p}^{\prime}$-DDD 值未检出, 说明这部分是典型 的有氧性生物降解. 此外, 吸附在表面沉积物中的 $\mathrm{p}, \mathrm{p}^{\prime}$-DDT 能发生光解反应, 即太阳光照射能加快 $\mathrm{p}, \mathrm{p}^{\prime}$-DDT 转变成 $\mathrm{p}, \mathrm{p}^{\prime}$-DDE 的速率 ${ }^{[23]}$.

\section{2 柱状样沉积物样 PAHs 含量的垂直分布特征}

2.2.1 PAHs 含量与垂直分布 本次样品中, 共检测 出 16 种优控 PAHs 中的 12 种, Ace、Flu、BaA 以及 IP 在所有样品中均未检出 (表 3). Pyr、Phe 和 Ant 的检测含量最高, 分别为 $17.1 \sim 67.1 \mathrm{ng} / \mathrm{g}(\mathrm{dw}$ ) (平均 $32.5 \mathrm{ng} / \mathrm{g}(\mathrm{dw}$ ) ) 、2.7 139.2 $\mathrm{ng} / \mathrm{g}(\mathrm{dw}$ ) (平均 $15.0 \mathrm{ng} /$ $\mathrm{g}(\mathrm{dw})$ ) 和 $3.6 \sim 34.4 \mathrm{ng} / \mathrm{g}(\mathrm{dw}$ ) (平均 $11.7 \mathrm{ng} / \mathrm{g}$ $(\mathrm{dw}))$. PAHs 总含量的垂直变化结果可见, 周溪湾 状沉积物中 PAHs 总量范围为 $41.3 \sim 384.0 \mathrm{ng} / \mathrm{g}$ $(\mathrm{dw})$, 平均为 $89.4 \mathrm{ng} / \mathrm{g}(\mathrm{dw})$, 分别出现在 ZX32 (62 64 cm) 处和 ZX1 $(0 \sim 2 \mathrm{~cm}$ ) 处 (图 3). 就表层沉 积物中 PAHs 含量而言 $(214 \sim 384 \mathrm{ng} / \mathrm{g}(\mathrm{dw})$, 平均 $299 \mathrm{ng} / \mathrm{g}(\mathrm{dw}))$, 与国内其它湖泊和河流相比处于 中等水平, 高于淮河 (淮南-蚌埠段, $5.4 \sim 78.3 \mathrm{ng} / \mathrm{g}$

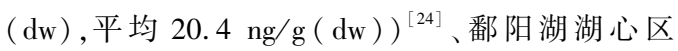
$\left(33.0 \sim 369 \mathrm{ng} / \mathrm{g}(\mathrm{dw})\right.$, 平均 $157 \mathrm{ng} / \mathrm{g}(\mathrm{dw})^{[8]}$ 和白 洋淀 (101 322 $\mathrm{ng} / \mathrm{g}(\mathrm{dw})$, 平均 $190 \mathrm{ng} / \mathrm{g}(\mathrm{dw}))^{[25]}$; 低于巢湖 (116 2832 ng/g ( dw), 平均 $899 \mathrm{ng} / \mathrm{g}$ $(\mathrm{dw}))^{[26]}$ 和太湖 $(259.1 \sim 1059.4 \mathrm{ng} / \mathrm{g}(\mathrm{dw})$, 平均 $580.5 \mathrm{ng} / \mathrm{g}(\mathrm{dw}))^{[27]}$.

根据 PAHs 总含量的垂直波动情况, 将其大致 分成两个阶段: 1990 年以前, PAHs 总含量变化起 伏不大, 总体在 $50 \sim 150 \mathrm{ng} / \mathrm{g}(\mathrm{dw})$ 范围内上下波 动, 最主要的是其波动情况与单个化合物 Pyr 含量 的波动保持了良好的一致性, 说明这阶段 PAHs 总 含量的变化主要受到 Pyr 含量的影响, 也说明这段 时期内 PAHs 的污染以 Pyr 为主. 1990 年至今, $\mathrm{PAHs}$ 总含量的波动情况与 Phe 含量保持明显的一 致性. 且自 2005 年之后, PAHs 总含量呈现急剧的 连续上升趋势, 远远超过往年的最高峰值, Phe 含 
量亦表现出明显增加, 而此时 Pyr 含量却相对减少, 说明近十几年来影响 PAHs 总含量增幅的主要因素是 Phe 的大幅释放,并且该区 PAHs 和 Phe 的释放源及其向湖内环境的输人量在不断增加, 并且有逐年上升的 趋势.

2.2.2 PAHs 组分与源解析 根据环数不同, 把所测的 PAHs 分为 $2 \sim 3$ 环、 4 环和 $5 \sim 6$ 环. 1990 年以前, 4 环 化合物比重 (34.0\% 56.1\%, 平均为 $44.4 \%$ ) 略高于 $2 \sim 3$ 环 ( $33.1 \% \sim 55.3 \%$, 平均为 $43.4 \%$ ), $5 \sim 6$ 环化合物 最少. 1990 年至今, 3 环比重明显上升占据主导 ( $39.6 \% \sim 83.0 \%$, 平均为 $64.2 \%$ ), 远远高于 4 环 $(11.7 \% \sim$ $48.8 \%$, 平均为 $25.3 \%$ ) 和 5 6 环 $(4.12 \% \sim 27.6 \%$, 平均为 $10.4 \%$ ) (图 4). 总的来说, 1990 年以前, 高分子量 化合物 (HMW) 所占的比重要高于低分子量化合物 ( LMW), 而近 20 年来, LMW 占 PAHs 的绝大部分. 一般 情况认为, LMW 主要来源于石油类产品、化石燃料的不完全燃烧或天然成岩过程, 而高温热解主要生成沸 点高、水溶性较低的 $\mathrm{HMW}^{[28]}$.
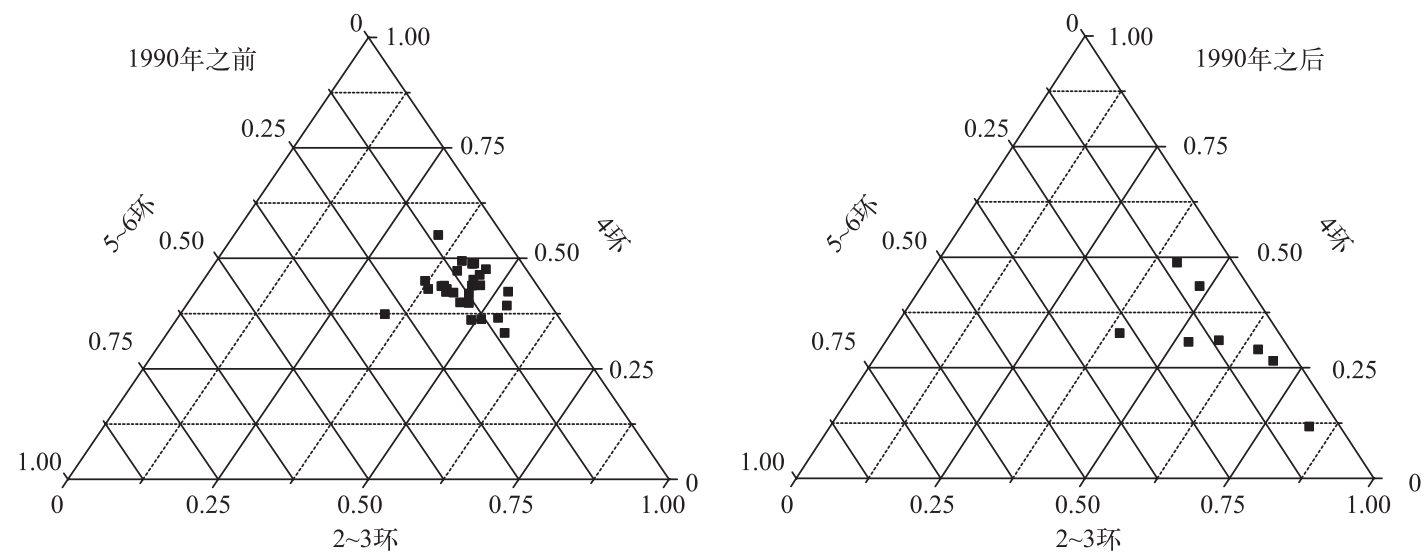

图 4 周溪湾柱状沉积物 PAHs 组分三角百分比图 *

Fig.4 Compositions of PAHs in sedimentary Zhouxi Bay core

为了进一步提高对周溪沉积物 PAHs 来源判 表 41990 年前 PAHs 主因子特征根与旋转后载荷矩阵* 定的精确度, 本研究又对 36 个样品中 PAHs 含量 进行主成分分析和多元线性回归(PCA/MLR), $K M O$ 值为 0.735 , 大于 $0.5, P$ 值 $($ sig. $=0.000)<$ 0.05 , 适合做主成分分析. 结果表明, 1990 年以前, 12 种可检出 PAHs 化合物的 3 个主成分累计方差 贡献率为 $77.0 \%$, 各组分的载荷矩阵值详见表 4. PC1 和 PC2 分别在 Fl、Phe、BkF 以及 Ant、Chr 上 具有较重载荷,同时在 Pyr 上具有中等载荷,这些 物质主要是煤燃烧的标记物 ${ }^{[6,32-34]}$. PC3 显示 Acy 在该因子上具有较重载荷, $\mathrm{BbF} 、 \mathrm{BghiP}$ 和 $\mathrm{BaP}$ 具 有重度和中度载荷, 这两类又分别是木材燃烧的 排放物和交通汽油排放的示踪剂 ${ }^{[6,29-31]}$. 因此, 1990 年以前该区主要的 PAHs 来源有燃煤源、木 材燃烧源以及交通污染源; 同时根据多元线性回 归法计算得出燃煤源是其中最主要的污染, 其贡 献率占总污染的 $75.1 \%$. 而 1990 年以后, 12 种可 检出 PAHs 化合物的主影响因子只有 2 个, 累积 方差贡献率为 $83.7 \%$,中高分子化合物与 PC1 有

Tab.4 Rotated component eigenvalues and loadings of PAHs before 1990

\begin{tabular}{lccc}
\hline & PC1 & PC2 & PC3 \\
\hline 特征根 & 5.6 & 2.4 & 1.2 \\
方差贡献率/\% & 30.1 & 24.1 & 22.8 \\
累积方差贡献率/\% & 30.1 & 54.2 & 77.0 \\
Nap & 0.627 & -0.298 & -0.154 \\
Acy & -0.188 & 0.295 & $\mathbf{0 . 7 9 6}$ \\
Fl & $\mathbf{0 . 7 8 5}$ & 0.443 & 0.254 \\
Phe & $\mathbf{0 . 8 3 5}$ & 0.319 & 0.028 \\
Ant & 0.124 & $\mathbf{0 . 8 2 9}$ & 0.438 \\
Pyr & 0.644 & 0.637 & 0.345 \\
Chr & 0.187 & $\mathbf{0 . 8 5 1}$ & -0.008 \\
BbF & 0.206 & -0.193 & $\mathbf{0 . 8 9 2}$ \\
BkF & $\mathbf{0 . 7 4 0}$ & 0.417 & 0.292 \\
BaP & 0.040 & 0.510 & 0.586 \\
DahA & $\mathbf{0 . 8 7 8}$ & 0.034 & -0.018 \\
BghiP & 0.196 & 0.353 & $\mathbf{0 . 6 8 8}$ \\
\hline
\end{tabular}

*字体加粗表示主因子上具有较重载荷的化合物. 


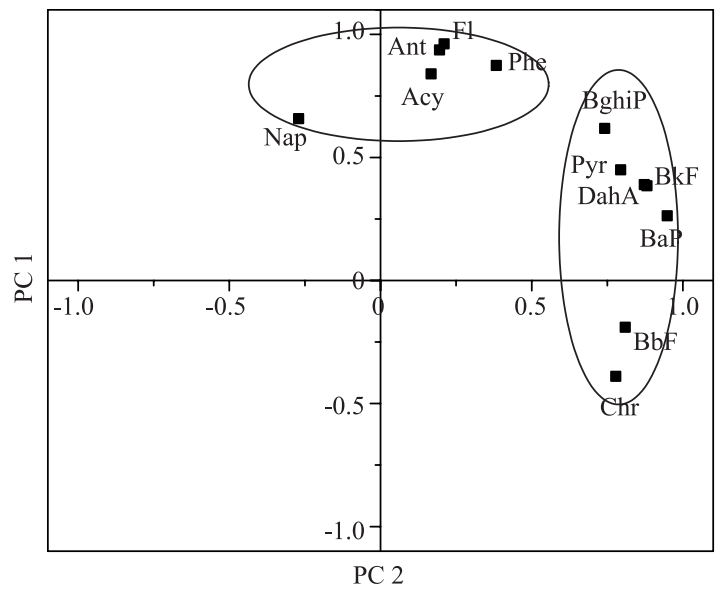

图 51990 年后多环芳烃主成分分析图

Fig.5 Principal component analysis of PAHs after 1990

较高的关联性, 相关性最高的是 $5 \sim 6$ 环的 $\mathrm{BaP}$ 、 $\mathrm{BkF} 、$ DahA 和 BghiP, 这类物质是典型的交通源汽 油和柴油排放的特征化合物 (图 5). BghiP 和 DahA 是交通汽油燃烧的示踪剂, 柴油机排放的废 气会带来 $\mathrm{BkF}^{[29-31]}$. 此外, 4 环的 Pyr 和 Chr 在 PC1 上也具有较重载荷, 他们是煤燃烧释放的中高环 化合物 ${ }^{[6,32-34]}$. 而中低环的 $\mathrm{Fl} 、$ Ant、Phe 和 Acy 在 PC2 上具有重度载荷, 这类化合物是煤燃烧的主 要产物; Nap 在该因子上显示中等载荷, 所表达的 $\mathrm{PAHs}$ 污染源为石油及相关产品的泄漏和挥发, 特 别是来自于水路运输的石油类泄漏 ${ }^{[35]}$. 所以, PC1 突出反映了表层样品中 PAHs 的来源为交通污染 源, PC2 反映了燃煤源和石油污染源; 同时结合多 元线性法得出交通污染源占总污染的 $67.5 \%$. 因 此,1990 年以后, 交通污染释放成为该区 PAHs 的 主要污染来源. 对比以上 2 个主成分分析结果可 显示, 近年来周溪湾地区 PAHs 的主要来源已经由煤燃烧释放转变为交通污染排放, 并伴随有石油类泄漏事 故的发生,这也是未来加大控制和治理该区 PAHs 污染的主要对象.

\section{3 结论}

1) 周溪湾柱状沉积物中 OCPs 总含量的垂直变化特征显示, 1950 年以前, 由全球长距离的迁移带来的 OCPs 导致其在沉积物中的残留量波动较大, 且含量偏高; 1950-1990 年 OCPs 总含量波动较小且残留量偏 低, 而到 1990-2000 年左右沉积物中 OCPs 残留量有个异常增高再降低的峰值与峰谷, 这种垂直变化规律 与我国生产和使用 OCPs 的实际情况相对应, 只是反映在沉积相上的残留量则向后推迟了 $10 \sim 20$ 年. 就表层 沉积样品而言, OCPs 残留量与国内其他地区相比总体是偏高的. HCHs、Chlordanes 以及 DDTs 是 OCPs 中主 要的检出物, 而 HCHs 和 DDTs 中又以 $\beta-H C H$ 和 $p, p^{\prime}-D D E$ 为主, 表明周溪湾地区目前已没有或鲜有新的 HCHs 和 DDTs 输人, 并且 Chlordanes 在环境中的存在时间也相对久远.

2) 周溪湾柱状沉积物中 PAHs 总含量的垂直变化特征显示, 1990 年以前 PAHs 总含量在 $50 \sim 150 \mathrm{ng} / \mathrm{g}(\mathrm{dw})$ 之间起伏, 受单个化合物 Pry 的影响大, 组分以 4 环化合物为主; 1990 年至今, PAHs 总含量急剧上升并且逐 年增加, Phe 成为影响 PAHs 的主要化合物, 3 环比重亦随即占据主导, 就表层沉积样品与国内其他地区相比 处于中等污染水平. 同时, 周溪湾地区 $\mathrm{PAHs}$ 的主要污染来源由以前的煤燃烧释放转化为交通污染排放为 主,并伴随有石油类泄漏事故的发生.

致谢:感谢鄱阳湖湖泊湿地观测研究站对本文野外样品采集提供的帮助.

\section{4 参考文献}

[ 1 ] Richardson BJ, Zheng GJ. Chlorinated hydrocarbon contaminants in Hong Kong surficial sediments. Chemosphere, 1999, 39(6): 913-923.

[ 2 ] Wu Y, Zhang J, Zhou Q. Persistent organochlorine residues in sediments from Chinese river/estuary systems. Environmental Pollution, 1999, 105(1): 143-150.

[ 3 ] XU Shifen, Jiang Xin, Feng Jian et al. Gas chromatographic method for the determination of organochlorine pesticides in suspended solids and sediments of the Yangtze River. Acta Scientiae Circumstantiae, 2000, 20(4) : 494-498(in Chinese with English abstract). [许士奋, 蒋新, 冯建等. 气相色谱法测定长江水体悬浮物和沉积物中有机氯农药的残留 量. 环境科学学报, 2000, 20(4): 494-498.]

[ 4 ] Baek S, Field R, Goldstone M et al. A review of atmospheric polycyclic aromatic hydrocarbons: sources, fate and behavior. Water, Air, and Soil Pollution, 1991, 60(3/4) : 279-300. 
[ 5 ] Khalili NR, Scheff PA, Holsen TM. PAH source fingerprints for coke ovens, diesel and, gasoline engines, highway tunnels, and wood combustion emissions. Atmospheric Environment, 1995, 29(4) : 533-542.

[ 6 ] Harrison RM, Smith D, Luhana L. Source apportionment of atmospheric polycyclic aromatic hydrocarbons collected from an urban location in Birmingham, UK. Environmental Science \& Technology, 1996, 30(3) : 825-832.

[ 7 ] Xu S, Liu W, Tao S. Emission of polycyclic aromatic hydrocarbons in China. Environmental Science \& Technology, 2006, 40(3): 702-708.

[ 8 ] Zhi H, Zhao ZH, Zhang L. The fate of polycyclic aromatic hydrocarbons (PAHs) and organochlorine pesticides (OCPs) in water from Poyang Lake, the largest freshwater lake in China. Chemosphere, 2015, 119(1): 1134-1140.

[ 9 ] Lu M, Zeng DC, Liao Y et al. Distribution and characterization of organochlorine pesticides and polycyclic aromatic hydrocarbons in surface sediment from Poyang Lake, China. Science of the Total Environment, 2012, 433: 491-497.

[10] Huang Yun, Zhong Huiming, Zhou Wenbin et al. Vertical distribution characteristics of organochlorinated pesticides in sedimentary Kangshan core of Lake Poyang. Jiangxi Science, 2009, 27(2) : 307-310(in Chinese with English abstract). [黄云, 钟恢明, 周文斌等. 鄱阳湖康山沉积物柱状样中有机氯农药的垂直分布特征及风险评估. 江西科学, $2009,27(2): 307-310$. ]

[11] Liu Xiaozhen, Zhou Wenbin, Hu Lina et al. Perpendicularity pollution characteristics of organochlorine pesticides in bottomland sediment of Poyang Lake area. Ecology and Environment, 2008, 17(4) : 1348-1353(in Chinese with English abstract). [刘小真, 周文斌, 胡利娜等. 鄱阳湖区洲滩底质有机氯农药的垂直污染特征. 生态环境, 2008, 17(4): 1348-1353.]

[12] Talking about Zhouxi in Poyang Lake. http://www.cndcta.com/news/201202/news_20120228115138. html, 2012-2-28. [ 鄱阳湖上话周溪. http://www.cndcta.com/news/201202/news_20120228115138.html, 2012-2-28.]

[13] Liu Nannan, Chen Peng, Zhu Shuzhen et al. Distribution characteratics of PAHs and OCPs in sediments of Liaohe River and Lake Taihu and their risk evaluation based on sediment quality criteria. China Environmental Science, 2011, 31(2) : 293-300 (in Chinese with English abstract). [ 刘楠楠, 陈鹏, 朱淑贞等. 辽河和太湖沉积物中 PAHs 和 OCPs 的分布 特征及风险评估. 中国环境科学, 2011, 31(2): 293-300.]

[14] Guo Zhangzhen, Meng Huisheng, Zhang Yuan et al. Polychlorinated biphenyl and organochlorine pesticides in surface sediments of Fenhe River: The concentration, sources and ecological risk. China Environmental Science, 2013, 33(4) : 714 721 (in Chinese with English abstract). [ 郭掌珍, 孟会生, 张渊等. 汾河表层沉积物 PCBs 和 OCPs 含量、来源及生态 风险. 中国环境科学, 2013, 33(4): 714-721.]

[15] Liu WX, Wei H, Qin N et al. The residues, distribution, and partition of organochlorine pesticides in the water, suspended solids, and sediments from a large Chinese lake (Lake Chaohu) during the high water level period. Environmental Science and Pollution Research, 2013, 20: 2033-2045.

[16] Zhao ZH, Zhang L, Wu JL et al. Distribution and bioaccumulation of organochlorine pesticides in surface sediments and benthic organisms from Taihu Lake, China. Chemosphere, 2009, 77(9): 1191-1198.

[17] Ye Chuanyong, Chen Suhua, Jiao Xingchun. Vertical distribution characteristics of organochlorine pesticides in sediments from Longkou of Lake Poyang. Rock and Mineral Analysis, 2009, 28(4) : 342-346 (in Chinese with English abstract). [叶传永, 陈素华, 焦杏春. 鄱阳湖龙口地区沉积物中有机氯农药的垂向分布特征. 岩矿测试, 2009, 28(4): 342-346.]

[18] Huang Jun, Yu Gang, Qian Yi. The problems of persistent organic pollutants in China and their research countermeasures. Environmental Protection, 2001, 11(3) :1-6(in Chinese with English abstract). [黄俊, 余刚, 钱易. 我国的持久性有 机污染物问题与研究对策. 环境保护, 2001, 11(3): 1-6.]

[19] Zhao Ci, Liu Xiaozhen, Zhou Lifeng et al. Pollution characteristics of organochlorinated pesticides in sediments from the middle and lower reaches of Ganjiang River. Ecology and Environmental Sciences, 2010, 19(10):2419-2424(in Chinese with English abstract). [赵慈, 刘小真, 周立峰等. 赣江流域中下游底泥中有机氯农药污染特征. 生态环境学报, 2010, 19(10):2419-2424. ]

[20] Hu JX, Zhu T, Li QL. Organochlorine pesticides in China. Developments in Environmental Science, 2007, $7: 159-211$.

[21] Bidleman, Jantunen LLM, Helm PA et al. Chlordane enantiomers and temporal trends of chlordane isomers in Arctic air. Environmental Science \& Technology, 2000,36: 539-544.

[22] Jiang YF, Wang XT, Jia Y et al. Occurrence, distribution and possible sources of organochlorine pesticides in agricultural 
soil of Shanghai, China. Journal of Hazardous Materials, 2009,170: 989-997.

[23] Huang Yun, Zhong Huiming, Liu Zhigang. Main organochlorine persistence characteristic in sediments of Lake Poyang. Jiangxi Science, 2010, 28(3) : 336-340( in Chinese with English abstract). [黄云, 钟恢明, 刘志刚. 鄱阳湖沉积物中 主要有机氯农药的残留特征. 江西科学, 2010, 28(3): 336-340.]

[24] Wang Qing, Ding Yi, Li Yucheng et al. Study on pollution characteristics of PAHS in sediments from the Huainan and Bangbu sections of Huaihe river. Journal of Suzhou University, 2008, 22(6) : 103-106( in Chinese with English abstract). [王庆, 丁毅, 李玉成等. 淮河淮南-蚌埠段沉积物中的多环芳烃污染性状研究. 宿州学院学报, 2008, 22(6): 103-106. ]

[25] Hu G, Luo X, Li F et al. Organochlorine compounds and polycyclic aromatic hydrocarbons in surface sediment from Baiyangdian Lake, North China: Concentrations, sources profiles and potential risk. Journal of Environmental Sciences, 2010, 22(2): 176-183.

[26] Ning Yi, Ke Yongchun, Deng Jiancai et al. Distribution and sources of polycyclic aromatic hydrocarbons (PAHs) in surface sediment in Lake Chaohu. J Lake Sci, 2012, 24(6) : 891-898( in Chinese with English abstract). DOI 10.18307/ 2012.0612. [宁怡, 柯用春, 邓建才等. 巢湖表层沉积物中多环芳烃分布特征及来源. 湖泊科学, 2012, 24(6): 891-898.]

[27] Wu Ting, Liu Guirong, Tian Yingze et al. Spatial distribution and source apportionment of PAHs in sediments from Lake Taihu. Journal of Agro-Environment Science, 2015, 34(1):124-129(in Chinese with English abstract). [武婷, 刘贵荣, 田瑛泽等. 太湖底泥多环芳烃分布及来源解析. 农业环境科学学报, 2015, 34(1):124-129.]

[28] Zhao ZH, Zhang L, Cai YJ et al. Distribution of polycyclic aromatic hydrocarbon ( PAH) residues in several tissues of edible fishes from the largest freshwater lake in China, Poyang Lake, and associated human health risk assessment. Ecotoxicology and Environmental Safety, 2014, 104: 323-331.

[29] Simcik MF, Eisenreich SJ, Lioy PJ. Source apportionment and source/sink relationships of PAHs in the coastal atmosphere of Chicago and Lake Michigan. Atmospheric Environment, 1999, 33(30) : 5071-5079.

[30] Lim LH, Harrison RM, Harrad S. The contribution of traffic to atmospheric concentrations of polycyclic aromatic hydrocarbons. Environmental Science \& Technology, 1999, 33(20) : 3538-3542.

[31] Chen Y, Sheng G, Bi X et al. Emission factors for carbonaceous particles and polycyclic aromatic hydrocarbons from residential coal combustion in China. Environmental Science \& Technology, 2005, 39(6) : 1861-1867.

[32] Lee RG, Coleman P, Jones JL et al. Emission factors and importance of PCDD/Fs, PCBs, PCNs, PAHs and PM10 from the domestic burning of coal and wood in the UK. Environmental Science \& Technology, 2005, 39(6) : 1436-1447.

[33] Larsen RK, Baker JE. Source apportionment of polycyclic aromatic hydrocarbons in the urban atmosphere: a comparison of three methods. Environmental Science \& Technology, 2003, 37(9) : 1873-1881.

[34] Zhu Ying, Wu Wenqian, Wang Junjun et al. Distribution, sources and ecological risks of polycyclic aromatic hydrocarbons in water-sediment system in Lake Small Baiyangdian. J Lake Sci, 2009, 21(5): 637-664(in Chinese with English abstract). DOI 10.18307/2009.0505. [ 朱樱, 吴文倩, 王军军等. 小白洋淀水-沉积物系统多环芳烃的分布、来源与生 态风险. 湖泊科学, 2009, 21(5): 637-664.]

[35] Li Zhe, Yu Na, Liu Ying et al. Distribution and sources of PAHs in surface sediments of a typical secondary tributary in the Three Gorges Reservoir Area. Sichuan Environment, 2012, 31(1): 17-23(in Chinese with English abstract). [李哲, 喻娜, 刘颖等. 三峡库区典型次级支流表层沉积物中 PAHs 的分布特征及来源分析. 四川环境, 2012, 31(1): 17-23.] 